\title{
Anesthetic management of a child with Down's Syndrome having atlanto axial instability
}

\author{
Bhattarai B, KulkarniA H, Kalingarayar S, Upadya MP \\ Department of Anesthesiology, Kasturba Medical College, Mangalore, India
}

\begin{abstract}
Down's syndrome is the most commonly encountered congenital anomaly in medical practice. These patients are of special concern to medical practice because of their associated problems with regard to respiratory, cardiovascular and other systemic problems. As these patients present for repeated surgeries like dental extraction, facial reconstruction and fixation of cervical spine, these patients pose challenges to the anesthesiologist because of their unique set of problems, namely atlantoaxial instability, small trachea, congenital heart disease and repeated chest infections due to lowered immunity. Their reactivity to inhalational anesthetics and atropine is variable. Here we present an interesting case report of a child with Down's syndrome who presented with atlantoaxial instability for MRI of cervical spine under general anesthesia.
\end{abstract}

Key Words: atlanto axial instability, down's syndrome, trisomy 21

\section{INTRODUCTION}

Multiple problems associated with Down's syndrome and involvement of various organ systems necessitates meticulous identification of these problems and careful handling of these children. In particular, attention to airway management and positioning with minimal movements of the cervical spine is essential, keeping in view the relatively high incidence of atlanto-axial instability(AAI). Although the incidence of $\mathrm{AAl}$ is high in patients with Down's syndrome, the reports of such complications and its management are very few in literature. Guidelines and recommendations in airway management and positioning during surgery for the patients are vague. We strongly feel the need to formulate evidence based guidelines and a cost effective preoperative screening tools to prevent cervical instability, since more Down's Syndrome children are being referred for anesthesia in the past two decades.
We describe herewith the management of a child of Down's Syndrome having atlanto axial instability who presented to us for Magnetic resonance imaging (MRI) of the neck.

\section{CASE REPORT}

A 12-year old child presented with complaints of progressive weakness of the left side of body. There was a history of fall few days prior. He had no other systemic complaints. There was history of surgery for duodenal atresia during neonatal period, which was uneventful. On examination, he was a moderately built, poorly nourished child, weighing $20 \mathrm{kgs}$. He had gross mental and physical retardation and mongoloid facies with upward slant of eyes, small nose with flat nasal bridge; he also had clinodactyly, siamian crease and sandal gap. He had a cervical collar in situ. Vital signs revealed heart rate of 90 beats per minute and 
Bhattarai et al. Anesthetic management of a child with Down's Syndrome having atlanto axial instability

blood pressure of $90 / 60 \mathrm{~mm}$ of $\mathrm{Hg}$. Examination of cardiovascular and respiratory systems revealed no gross abnormality. Nervous system examination revealed generalized hypotonia and left sided weakness with muscle power of 2/5 in left upper and lower limbs. On airway examination, the child had macroglossia with prominent upper incisors and there was limited neck mobility due to cervical collar. Routine hematologic tests were within normal limits. Previous echocardiogram done during his infancy revealed presence of patent ductus arteriosus whereas a recent one was normal. X-ray of cervical spine showed dislocation of atlanto axial joint. There had been a previous attempt at performing the MRI scan under oral sedation, and was unsuccessful. See fig 1

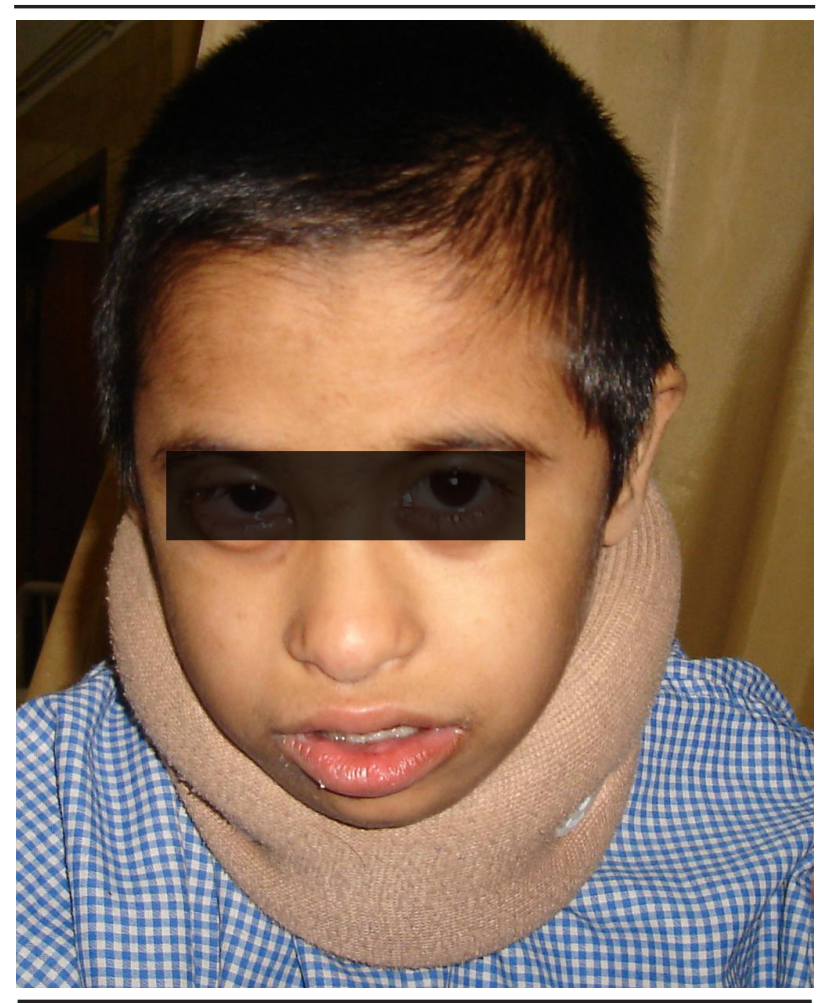

Figure 1. Child with Downs syndrome with cervical collar in situ making airway assessment difficult.

The child was shifted to MRI suite and ECG and pulse oximeter were connected. Induction of anesthesia was done with inhalation technique, using oxygen in $50 \%$ nitrous oxide, and progressively increasing concentration of halothane up to $3 \%$. Keeping in mind the possibility of difficult airway, need for an immobile neck and also possible tracheal stenosis in these children, a pediatric fibrescope was kept ready for use. Once good depth was obtained and satisfactory ventilation possible, neuromuscular blockade was achieved with atracurium $10 \mathrm{mg}$. A size 2 LMA was inserted. A bite block was placed. All the time, the neck was maintained in neutral position by keeping the soft cervical collar in place. Anesthesia was maintained with halothane in nitrous oxide and oxygen. Intraoperatively there was a single episode of frequent ventricular ectopics which was treated with lignocaine $30 \mathrm{mg}$. Towards the end of procedure, inhalation anesthetic was discontinued, nitrous oxide turned off and residual neuromuscular blockade was reversed with neostigmine and glycopyrolate. LMA was removed after the child was fully awake. A repeat neurologic examination was performed which revealed same findings as preprocedure. The child later underwent cervical spine stabilization.

\section{DISCUSSION}

Down's syndrome, also known as trisomy 21, is the commonest of congenital anomalies, occurring in 1 out of every 800 live births. ${ }^{1}$ The syndrome was first described by $\mathrm{Dr}$ John Langdon Down in 1866. Since 1866, when Down described the clinical features of the syndrome characterized by an extra chromosome 21 and a wide spectrum of phenotypic consequences, medical care has developed leaps and bounds and today life expectancy of a Down's child has increased dramatically. It is the best known chromosomal disorder in man. There is an extra copy of chromosome 21 , which affects all the organ systems and results in a wide spectrum of phenotypic consequences. This extra copy of the proximal part of $21 \mathrm{q} 22.3$ appears to result in the typical phenotype, mental retardation, hand anomalies and heart defects. There is increase in the incidence of Down's syndrome if the maternal age exceeds 35, exposure to pesticides or electromagnetic fields, exposure to anesthetic agents, drinking alcohol and caffeine-containing beverages.

As life expectancy has increased in these children because of better medical care, increasing number of them are being referred for surgery and require anesthesia for correction of cardiac defects, ENT, ophthalmologic, gastrointestinal and radiological procedures.

Craniocervical instability, most commonly atlantoaxial instability is the result of generalized ligamentous laxity, involving any of the three ligaments of the $\mathrm{C}_{1} \mathrm{C}_{2}$ joint. It can be acquired or precipitated by upper respiratory infections. Rotation of the head may result in $C_{1} C_{2}$ subluxation. An anterior arch of atlas to the dens of axis distance, anterior atlantodental interval (AADI), of greater than $4.5 \mathrm{~mm}$ indicates abnormal instability. The incidence of AAI varies widely; studies with roentgenograms in Down syndrome (DS) show an incidence of $20 \%$, while studies with plain films and CT images have a $50 \%$ incidence, symptomatic in 1 $2 \%$.These $20 \%$ of child who do not show the signs of atlanto axial instability is known as asymptomatic atlantoaxial instability. 
Bhattarai et al. Anesthetic management of a child with Down's Syndrome having atlanto axial instability

Considerable controversy exists regarding the screening of AAl in these patients. In 1984, AAP recommended restriction of contact sports if the AADi is greater than $4.5 \mathrm{~mm}$ and routine radiography between the ages of 3 and 5 yrs. But in 1995, AAP retracted their previous recommendations and released a revised statement that "lateral neck $X$ rays are of potential but unproven value in detecting patients at risk for developing spinal cord injury"

General anesthesia has the potential for subluxation of the joint, during laryngoscopy, positioning and transport. Various reports caution against forceful flexion of the neck, hyperextension during laryngoscopy; recommend strapping the patient to OR table and turning side to side for myringotomies. It is advisable to treat the patient with CS precautions for urgent surgeries and symptomatic instability.

For the diagnosis of atlantoaxial instability, lateral radiographs of cervical spine in flexion, extension and neutral position are sufficient. Atlanto-dental interval of $3-5 \mathrm{~mm}$ is considered to be borderline; where as the values of $12-13 \mathrm{~mm}$ is usually associated with symptoms. $^{2}$

For all the above reasons, positioning of head and neck during anesthetic management may place the spinal cord at risk if ligamentous instability is present, so the patients are recommended to undergo radiological evaluation of cervical spine before anesthesia. ${ }^{3}$

In addition, laxity of other joints such as fingers, thumb, elbows \& knees tends to correlate well with presence of atlantoaxial dislocation. ${ }^{4}$

The signs and symptoms of atlantoaxial instability include easy fatigability, difficulty in walking, abnormal gait, neck pain limiting neck mobility, torticollis, incoordination and clumsiness, sensory deficits, spasticity, hyperreflexia. These signs and symptoms remain stable for months or years; occasionally they progress and may result in hemiplegia, quadriplegia and death.

Due to the risk associated with Downs's syndrome, perioperative care needs to focus on the likelihood of cervical spine instability. Great care must be taken to maintain the neck in neutral position. If atlantoaxial instability is revealed in the radiographic examination, the child should be referred to a neurosurgeon and orthopedic surgeon for further evaluation and stabilization of cervical spine is necessary before any elective surgery is undertaken. ${ }^{3}$

LMAs have proven to be a valuable tool in airway management in these children with neck in neutral position. Although some authors have reported up to $95 \%$ success rate in insertion of LMA in neutral position in child with atlanto axial instability ${ }^{5}$, but Sahin ST et al concluded that fibreoptic laryngoscopy is the more suitable technique when cervical movement is not desired. ${ }^{6}$ However, insertion of endotracheal tube over the fibreoptic bronchoscope does involve neck movement.

Other problems associated with these patients are cardiac anomalies, which includes endocardial cusion defects $(40 \%)$, atrial septal defect $(27 \%)$, persistence of ductus arteriosus $(12 \%)$ and tetralogy of Fallot (8\%). Our patient had patient ductus arteriosus in childhood echocardiography but recent echocardiography was normal.

There is increased incidence of congenital subglottic stenosis, tracheal stenosis, and tracheal bronchus in these children?

There is increased incidence of respiratory complications in children with Downs's syndrome. Upper and lower airway problems exist in this subset of the population which is attributed to hypotonia, relative obesity, cardiac disease, small upper airway, degree of pulmonary hypoplasia, congenital anomalies of airway. This has also been related to immune deficiency and cardiac anomaly. This may also result in pulmonary arterial hypertension. As a result inadvertent hypoxemia, acidosis and hypercarbia are detrimental to these patients.

There is also increased incidence of pulmonary infections and relative frequency of positive hepatitis-associated antigens. This may also be due to thymus dependent immune system depression than humoral in children with Downs's syndrome. ${ }^{8}$ Strict aseptic precautions should be taken during intravenous and central venous cannulation and central venous catheter to be removed as soon as possible after the surgery.

Both exaggerated mydriatic response to ocular atropine and an increased response of heart rate to parenteral atropine have been documented ${ }^{9}$. So we used glycopyrrolate instead of atropine during reversal of the effect of neuromuscular blocking agent.

Decreased CNS catecholamine levels have been demonstrated in children with Down's syndrome, which may result in decrease in MAC of inhaled anesthetic agents. ${ }^{10}$

Gastro-esophageal reflux disease (GERD) is more prevalent in children with Down's syndrome. The symptoms to be assessed preoperatively include vomiting, oesophagitis, respiratory symptoms like apnea, wheezing and aspiration pneumonia. ${ }^{11}$ 
Bhattarai et al. Anesthetic management of a child with Down's Syndrome having atlanto axial instability

Anesthesiologists are sometimes involved in patient care during MRI as the quality of the image depends in part on the patient remaining immobile. In addition to the unique problems of an MRI suite like patient's inaccessibility, requirement of compatible monitors and machine and propensity to hypothermia, meticulous attention is recommended in protecting CS during transport and positioning the anesthetized child inside the MRI tube. In a retrospective study by Malviya et al they concluded that general anesthesia is a safe alternative to facilitate MRI in children with a higher ASA status and those with a history of failed sedation ${ }^{12}$.

\section{REFERENCES}

1. Yang Q, Rasmussen SA, Friedman JM. Mortality associated with Downs's syndrome in USA from 1983-1997: a population based study. Lancet. 2002 Mar 23;359(9311):1019-25.

2. Alvarez N. Atlantoaxial Instability in individuals with Down syndrome. E medicine [serial online] 2008 Dec 8 [Cited 2009 Jan 26]; 1(1) [5 screens]. Available from: URL: http:// emedicine.medscape.com/article/1180354-overview

3. Pushchel SM, Findley TW, Furia J, Gallaghar PL, Scola FH, Pezzullo JC. Atlantoaxial instability in Downs's syndrome: Roentgenographic, Neurological and Somatosensory evoked potential study J Pediatr. 1987 Apr;110(4):515-21.

4. Martin K, Creighton RE, Steward DJ. Anesthetic Considerations in Down's syndrome: Experience with 100 Patients and a Review of Literature. Can Anaesth Soc J. 1982 Nov;29(6):593-9.

5. Brimacombe J, Berry A. Laryngeal mask insertion. A comparison of the standard versus neutral position in normal patients with a view to use in cervical instability. Anaesthesia. 1993 Aug;48(8):670-1.

6. Richardson MA, Cotton RT. Anatomic abnormalities of the pediatric airway. Pediatr Clin North Am. 1984 Aug;31(4):82134.
7. Sahin A, Salman MA, Erden IA, Aypar U. Upper cervical vertebrae movement during intubating LMA, fibreoptic and direct laryngoscopy: a video fluoroscopic study. Eur J Anaesthesiol. 2004 Oct;21(10):819-23.

8. Levin S, Schlesinger M, Handzel Z, Hahn T, Altman Y, Czernobilsky B, Boss J. Thymic deficiency in Down's syndrome. Pediatrics. 1979 Jan;63(1):80-7.

9. Berg JM, Brandon MWG, Kirman BH. Atropine in mongolism. Lancet. 1959 Sep 26;2(7100):441-2.

10. Keele DK, Richards C, Brown J, Marshall J. Catecholamine metabolism in Down's syndrome. Am J Ment Defic. 1969 Jul; 74(1):125-9.

11. Cohen WI. Healthcare Guidelines for child with downs syndrome. Downs Syndr Q 1996 Sep;12:1-11.

12. Malviya S, Voepel-Lewis T, Eldevik OP, et al. Sedation and general Anesthesia in children undergoing MRI and CT: adverse effects and outcomes. Br J Anesth 2000 June; 84: 743 748 . 